\title{
Improving the shape of the cross-correlation function for leak detection in a plastic water distribution pipe using acoustic signals
}

\author{
Yan Gao $^{\mathrm{a}, *}$, Michael J. Brennan ${ }^{\mathrm{b}}$, Yuyou Liu ${ }^{\mathrm{c}, \mathrm{d}}$, Fabrício C.L. Almeida ${ }^{\mathrm{e}}$, Phillip F. Joseph ${ }^{\mathrm{f}}$ \\ ${ }^{a}$ Key Laboratory of Noise and Vibration Research, Institute of Acoustics, Chinese Academy of Sciences, Beijing 100190, China \\ ${ }^{\mathrm{b}}$ Department of Mechanical Eng., State University of São Paulo (UNESP), Ilha Solteira Campus, Av. Brasil Centro, 56, 15385-000 Ilha Solteira, Brazil \\ ' Beijing Municipal Institute of Labour Protection, Beijing 100054, China \\ ${ }^{\mathrm{d}}$ AECOM Infrastructure \& Environment UK Limited, London SW19 4DR, UK \\ ${ }^{\mathrm{e}}$ Department of Biosystem Eng., State University of São Paulo (UNESP), Tupã Campus, Av. Rua Domingos da Costa Lopes, Jardim Itaipu, 780, 17602-496 Tupã, Brazil \\ ${ }^{\mathrm{f}}$ Institute of Sound and Vibration Research, University of Southampton, Southampton SO17 1BJ, UK
}

\section{A R T I C L E I N F O}

\section{Article history:}

Received 10 December 2016

Received in revised form 22 May 2017

Accepted 25 May 2017

Available online 4 June 2017

\section{Keywords:}

Leak detection

Water pipe

Cross-correlation function

Time delay estimation

Resonance

\begin{abstract}
A B S T R A C T
This paper is concerned with time delay estimation for the detection of leaks in buried plastic water pipes using the cross-correlation of leak noise signals. In some circumstances the bandwidth of over which the signal analysis can be conducted is severely restricted because of resonances in the pipe system, which manifest themselves as peaks in the modulus of the power spectral and cross spectral densities, and deviations from straight-line behaviour in the phase of the cross spectral density. The result can be a crosscorrelation function in which it is difficult to estimate the time delay accurately. This paper describes a procedure in which the shape of the cross-correlation function can be significantly improved, resulting in an unambiguous and clear estimate of the time delay. The frequency response function(s) of the resonator(s) responsible for the resonance effects are first determined and then the data is processed using the model(s) of the resonators to remove these effects. This enables more signal processing to be conducted, potentially over a much wider bandwidth, further improving the shape of the cross-correlation function. The process is illustrated in this paper using hydrophone measured data at a leak detection facility. The current limitation in the process is that it is carried out manually, which could potentially restrict its application in practical acoustic correlators. The challenge now is to develop an algorithm to carry out the procedure automatically.
\end{abstract}

(c) 2017 Elsevier Ltd. All rights reserved.

\section{Introduction}

Water distribution networks are of paramount importance for maintaining a substantive modern life and economic growth. Underground pipes are susceptible to leakage, due to excavation damage, sabotage, deterioration and aging. Water leakage is a subject of increasing concern across the world because of the potential danger to public health, economic constraints, environmental damage and wastage of energy. Acoustic based leak detection techniques have been developed over the past 30 years, and are in common use in water distribution networks $[1,2]$. One such technique uses cross-correlation of measured leak noise signals to determine the difference in arrival times (time delay) between acoustic/vibration signals measured either side of a water leak. Together with the

\footnotetext{
* Corresponding author.

E-mail address: gaoyan@mail.ioa.ac.cn (Y. Gao).
}

knowledge of the speed of noise propagation, this is then used to determine the location of the leak.

Time delay estimation (TDE) is of great interest in many engineering fields, such as direction finding, source localization, and velocity tracking [3]. It can be carried out in the time and/or frequency domains [4,5]. Most commercial leak noise correlators utilize the basic cross-correlation (BCC) function via the Fast Fourier transform for TDE. In plastic pipes, measured water leak noise mostly occurs at low frequencies, below about $200 \mathrm{~Hz}$, although this can be higher for leaks close to a measurement point. The reason for this predominantly low frequency content, is that a plastic pipe essentially acts as an acoustic low-pass filter, which degrades the TDE procedure using the conventional BCC, in particular in the case of a poor signal-to-noise ratio [6,7]. To overcome this problem, Gao et al. [4] applied a pre-whitening process, using appropriate frequency weighting functions to improve the resolution of the time delay estimate. One particularly effective correlator uses only the phase spectrum, setting the modulus of the cross-spectrum to unity over the frequency range of analysis. This is the so-called 
phase transform (PHAT) correlator [4], which gives equal weighting to all frequencies, effectively increasing the bandwidth over which the time delay is estimated, compensating to some extent for the low-pass filtering properties of the pipe [7]. The prewhitening approach can, however, cause errors in the time delay estimate if there are additional phase shifts in the measurements caused by the dynamic behaviour of the pipe system. Such phase shifts have been observed by Gao et al. [6] in hydrophone measured data and by Almeida [8] in accelerometer measured data. The associated increase in the modulus of the cross-spectrum at the frequencies where there is an additional $90^{\circ}$ of phase shift suggest that the dynamics is due to resonance behaviour. The resonance behaviour is highly undesirable from the perspective of leak detection, as it can significantly reduce the bandwidth over which the BCC extracts time delay information.

The aim of this paper is to describe a method to remove the additional phase shifts due to the resonances in the system, so that a much wider bandwidth can be utilized, thus improving the shape of the cross-correlation function, making it easier to determine the time delay estimate. To achieve this, the ROTH correlator is used (as this is based on the frequency response function (FRF) of the pipe system [4,9]), which facilitates the visualization of the resonance effects. Using a model of a resonator capable of capturing the additional phase shift behaviour observed in measured data, these phase shifts are subsequently removed leaving only the phase spectrum due to time delay. This process is illustrated using some experimental data measured on an actual PVC water pipe.

\section{Problem statement}

Fig. 1 depicts a typical arrangement for water leak detection based on cross-correlation. Pipe fittings such as meters, values and fire hydrants are used as access points for the installation of the acoustic/vibration sensors such as hydrophones and accelerometers. The leak generates broadband noise, which propagates along the pipe, and the difference in the arrival times of the noise at the sensors (time delay) is used to determine the position of the leak. This is given by Ref. [6],

$d_{1}=\frac{d-c T_{0}}{2}$

where $c$ is the propagation speed of the leak noise (wave speed); $d$ is the distance between the sensors; and $T_{0}$ is the time delay estimate.
In many cases the wave speed is estimated from tables, but it can also be measured in-situ [10]. The time delay $T_{0}$ is estimated from the peak in the cross-correlation function between the two measured signals $x_{1}(t)$ and $x_{2}(t)$, which is given by Ref. [4]

$R_{x_{1} x_{2}}(\tau)=\frac{1}{2 \pi} \int_{-\infty}^{+\infty} W(\omega) e^{j \omega \tau} d \omega$,

where $W(\omega)=\Psi(\omega) S_{x_{1} x_{2}}(\omega)$, in which $\Psi(\omega)$ is given in Table 1 for the three correlators considered in this paper; $j=\sqrt{-1}$; $S_{x_{1} x_{2}}(\omega)=\left|S_{x_{1} x_{2}}(\omega)\right| e^{j \phi_{x_{1} x_{2}}(\omega)}$ is the cross spectral density (CSD) function, in which $\left|S_{x_{1} x_{2}}(\omega)\right|$ and $\phi_{x_{1} x_{2}}(\omega)$ are the modulus and phase spectra between the signals $x_{1}(t)$ and $x_{2}(t)$ respectively; and $\omega$ is circular frequency. The time delay estimate can also be determined from the frequency domain data by Ref. [5]

$T_{0}=-\frac{\sum_{i=1}^{N / 2}\left|W_{i}\right| \phi_{i} \omega_{i}}{\sum_{i=1}^{N / 2}\left|W_{i}\right| \omega_{i}^{2}}$,

where the subscript $i$ denotes the variable at the $i$-th frequency. Thus, the peak in the correlation function corresponds to the gradient of a straight line which is fitted to the measured phase in a weighted least squares sense.

Measured signals from a pipe rig, specially constructed for water leak detection at the National Research Council campus in Canada, are used to illustrate some issues. The description of the test site and measurement procedures are given in [2,11], and a plan of the site is shown in Fig. 2. Noise from a leak, an illustration of which is shown in Fig. 3(a), was measured using hydrophones, one of which is shown in Fig. 3(b). The distance between the measurement points was $102.6 \mathrm{~m}$, and the distance of the upstream measurement point from the leak was $73.5 \mathrm{~m}$. The signals of $66 \mathrm{~s}$ duration were each passed through an anti-aliasing filter with the cut-off frequency set at $200 \mathrm{~Hz}$ and then digitized at a sampling frequency of $500 \mathrm{~Hz}$.

Some processed data from the two leak noise signals are shown in Fig. 4. These were determined using a 1024-point FFT, a Hanning window (with 50\% overlap) and spectral averaging. Fig. 4(a) shows the power spectral densities (PSDs) of the two measured signals. Apparently, the PSD plots show that the signals attenuate with frequency and distance from the leak. It is also clear that there is resonance behaviour, which is evident in each measurement. The PSD

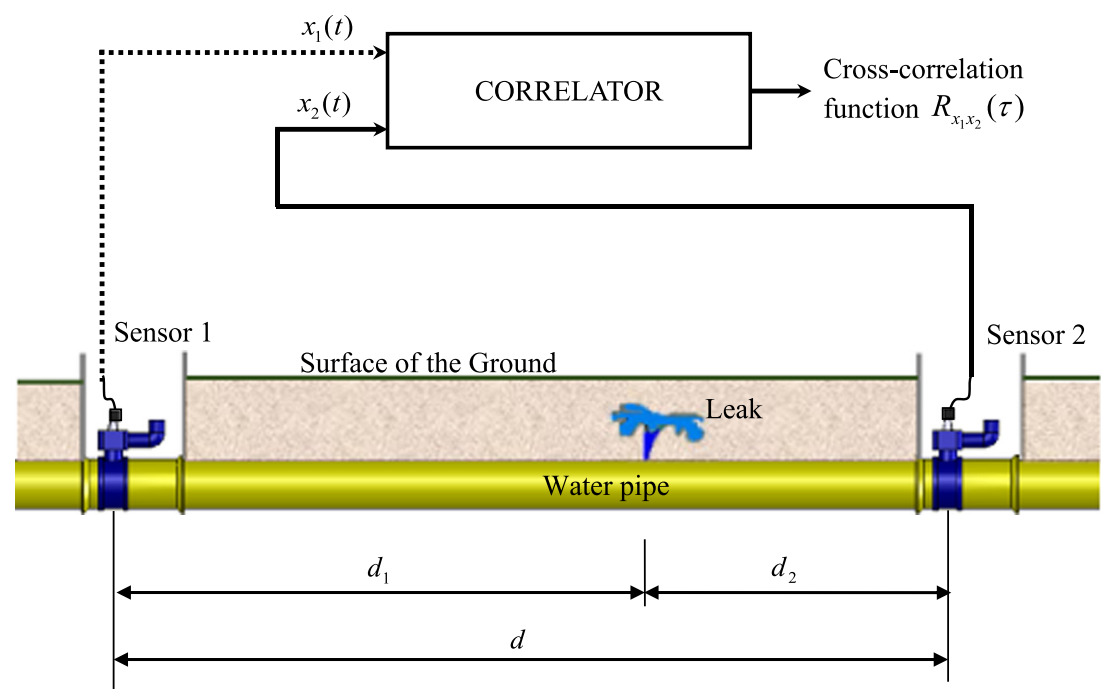

Fig. 1. Schematic of the process of leak detection in a buried water pipe using acoustic signals with a leak bracketed by two sensors. 
Table 1

The weighting functions used in the correlators.

\begin{tabular}{lll}
\hline Correlator & $\Psi(\omega)$ & $\left|W_{x_{1} x_{2}}(\omega)\right|$ \\
\hline BCC & 1 & $\left|S_{x_{1} x_{2}}(\omega)\right|$ \\
PHAT & $\frac{1}{\left|S_{x_{1} x_{2}}(\omega)\right|}$ & 1 \\
ROTH & $\frac{1}{S_{x_{1} x_{1}}(\omega)}$ or $\frac{1}{S_{x_{2} x_{2}}(\omega)}$ & $\frac{\left|S_{x_{1} x_{2}}(\omega)\right|}{S_{x_{1} x_{1}}(\omega)}$ or $\left|W_{x_{2} x_{1}}(\omega)\right|=\frac{\left|S_{x_{2} x_{1}}(\omega)\right|}{S_{x_{2} x_{2}}(\omega)}$ \\
\hline
\end{tabular}

with the resonant peak at $56 \mathrm{~Hz}$ is for the measurement downstream, which is closer to the leak, and consequently has a higher value than the PSD with a peak at $83 \mathrm{~Hz}$, which is upstream of the leak. At frequencies greater than the resonance frequencies, the signal levels attenuate even more rapidly with frequency, and this has an important effect on the behaviour of the correlators. This is discussed in Section 4. The CSD between the two signals is shown in Fig. 4(b). The two resonant peaks are evident in this plot as is the attenuation due to the low-pass filtering properties of the pipe. The coherence is plotted in Fig. 4(c), which shows that the bandwidth over which there is a reasonable linear relationship between the two signals is between about $5 \mathrm{~Hz}$ and $90 \mathrm{~Hz}$. Outside this bandwidth, the signals are contaminated with high degree of uncorrelated noise. The bandwidth $5-90 \mathrm{~Hz}$ is thus chosen for subsequent analysis, and this is shown on the graphs in Fig. 4.

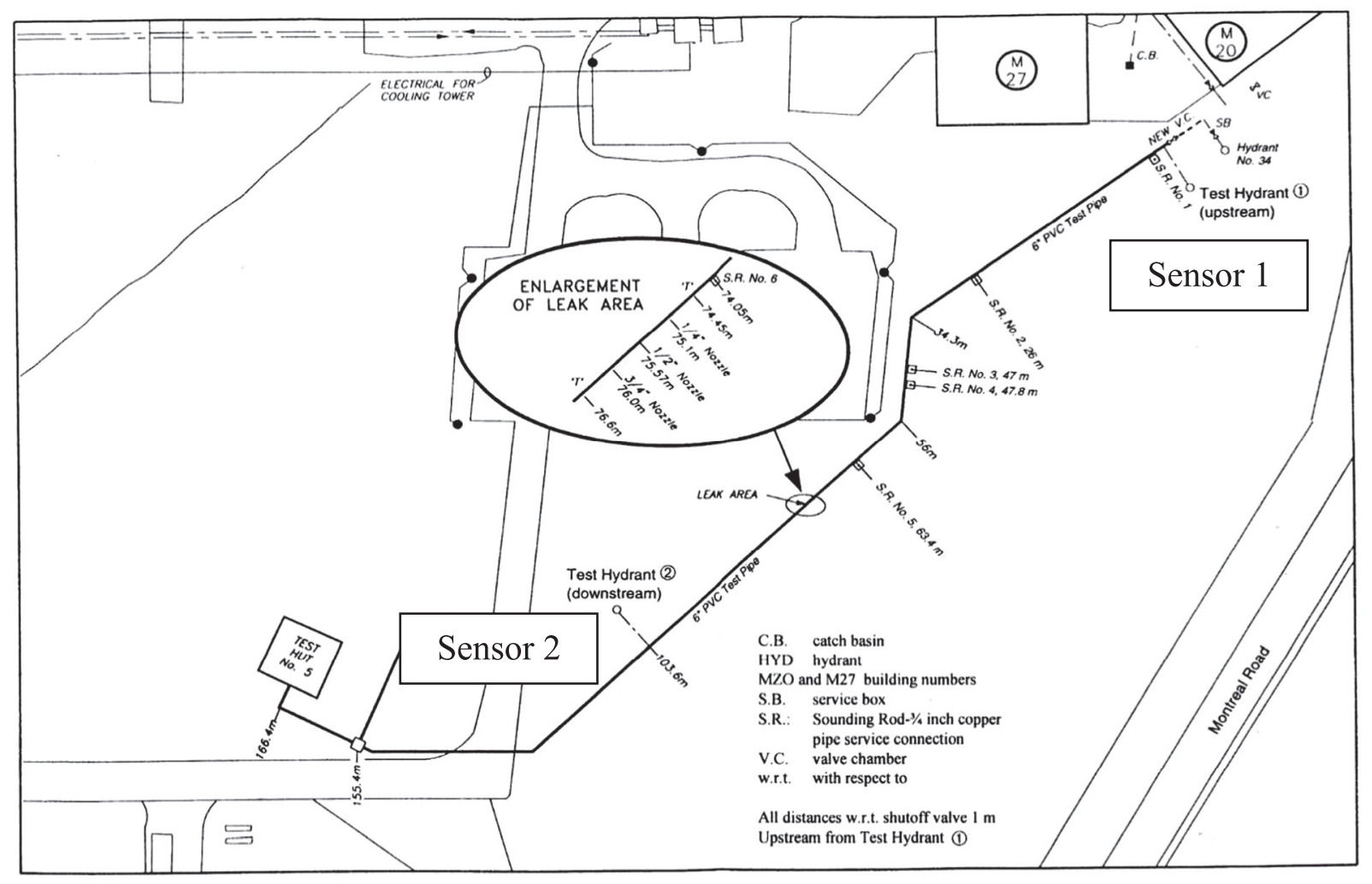

Fig. 2. Plan view of the experimental leak detection facility at the NRC, Canada [11].

(a)

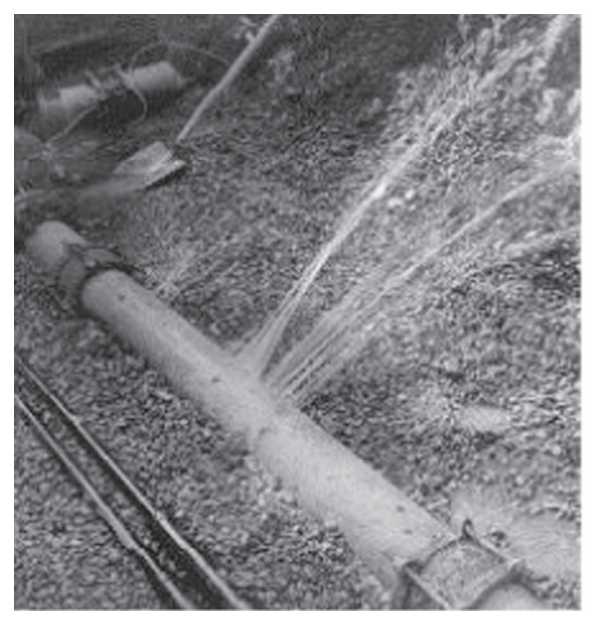

(b)

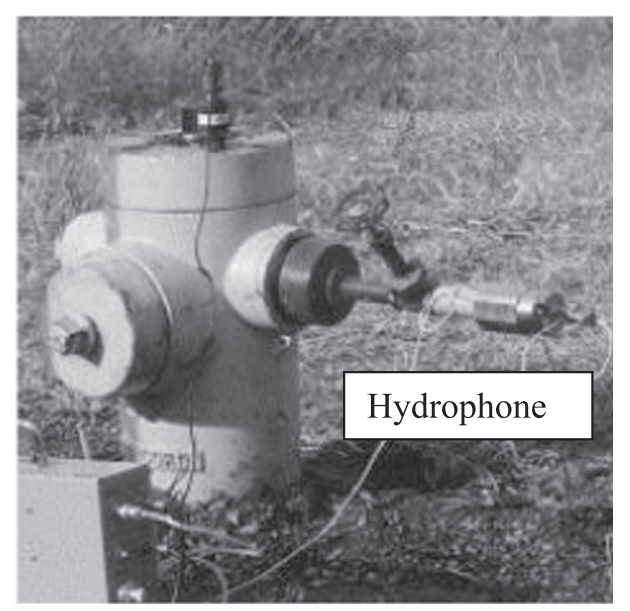

Fig. 3. Photographs illustrating a leak and a measurement point: (a) illustrative picture of a leak before backfill; (b) one of the measurement positions [2]. 
The phase of the CSD is shown in Fig. 4(d). The measured data is plotted as a blue solid line, and overlaid on this is a straight line, which is what the (ideal) phase would be if the pipe was infinite in length and located in an infinite medium, so that there were no reflections or resonance behaviour. The ideal phase was determined by multiplying the estimated time delay (see Section 4), the angular frequency and minus one. The resonances at $56 \mathrm{~Hz}$ and $83 \mathrm{~Hz}$ are evident, where the phase first deviates from the straight line by $\pi$ rads and then returns to the straight line respectively. Also evident are the deviations from the phase at very low frequencies, less than about $20 \mathrm{~Hz}$, which are due to acoustic reflections in the pipe system [12].

The modulus of the CSD attenuates rapidly with frequency because of (a) the damping properties of the pipe system, and (b) the resonances. As a result, the bandwidth of the signals used for TDE is restricted to low frequencies. This is particularly relevant for the BCC function in which the weighting function,

(a)

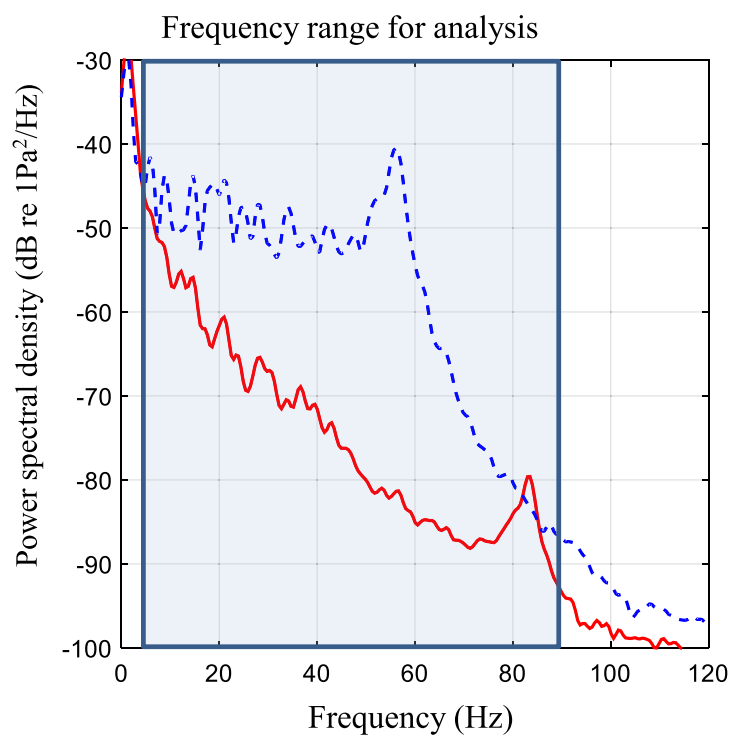

(c)

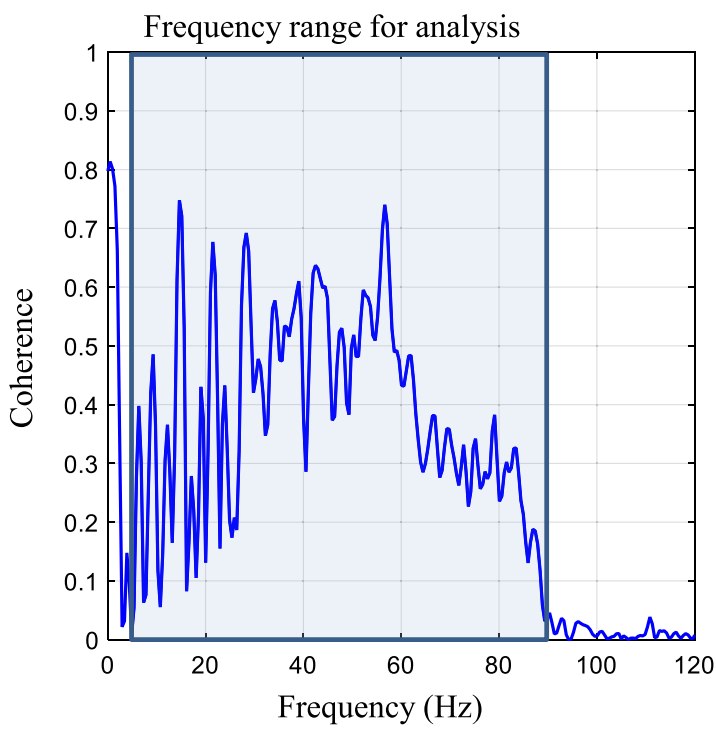

$\left|W_{x_{1} x_{2}}(\omega)\right|=\left|S_{x_{1} x_{2}}(\omega)\right|$, which suffers significant attenuation at high frequencies as can be seen in Fig. 4(b). If the PHAT correlator is used to overcome the adverse effects of a small weighting function at high frequencies due to the damping in the pipe system, then the phase shifts due to the resonances can cause an error in the TDE [8]. Thus, there is the motivation to remove the resonance effects in the measured signals, and this is further developed in the next section.

\section{Process for improving the shape of the cross-correlation function}

Conceptually, the passage of a leak noise signal from the leak to the sensor can be thought of as passing through a pipe filter and then a resonator. The resonance effects can thus be removed if the FRF for each resonator is known. If it is assumed that there is

(b)

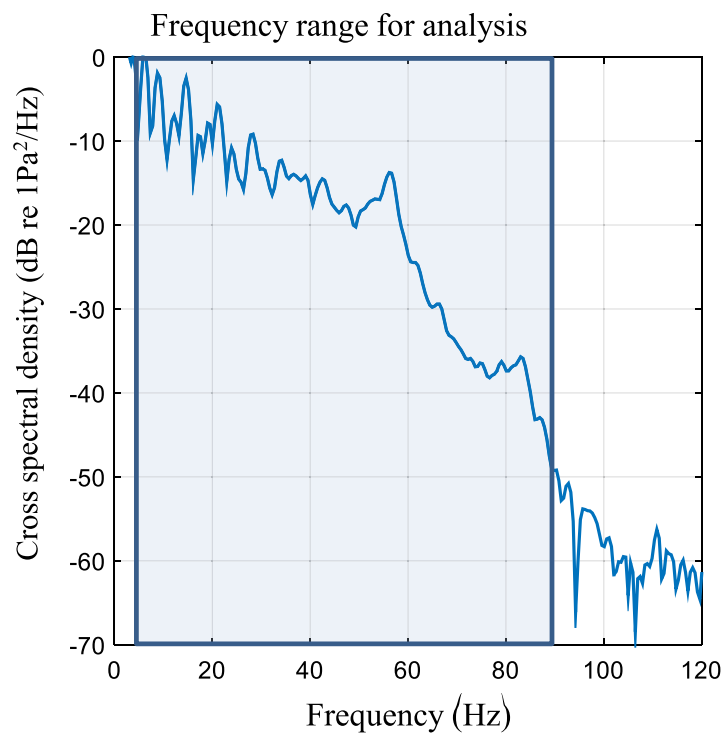

(d)

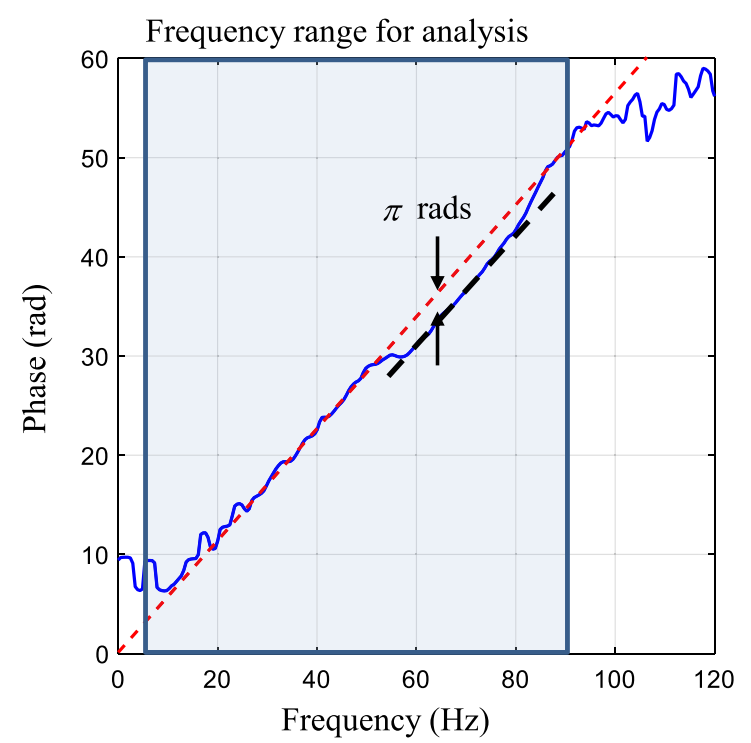

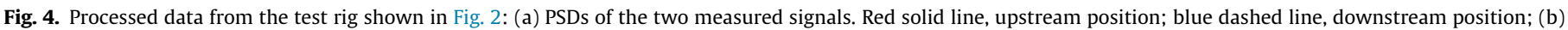

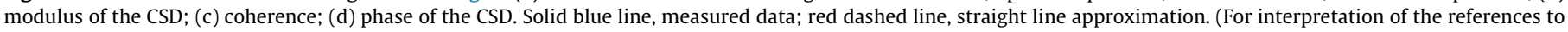
colour in this figure legend, the reader is referred to the web version of this article.) 
a resonator at sensor 1 with FRF $D_{1}$ and a resonator at sensor 2 with FRF $D_{2}$, then provided that the parameters of the resonators are known, the effects of the resonances can be removed from the measured data by simply multiplying $W(\omega)$ in Eq. (2) by $D_{1} / D_{2}$ for $S_{x_{1} x_{2}}(\omega)$ and by $D_{2} / D_{1}$ for $S_{x_{2} x_{1}}(\omega)$, where the subscripts denote the resonators at sensors 1 and 2 respectively. The FRF of a generic resonator is given by

$D(\omega)=\frac{\omega_{n}^{2}+j 2 \zeta \omega \omega_{n}}{\omega_{n}^{2}-\omega^{2}+j 2 \zeta \omega \omega_{n}}$

where $\omega_{n}$ and $\zeta$ are the natural frequency and damping ratio of the resonator respectively.

To determine the properties of the resonators, it is preferable to use the ROTH correlator, as this is similar to a conventional measured FRF as can be seen in the final column of Table 1 . This means the ROTH correlator will contain both resonances and antiresonances if they exist (which are the features related to the pipe dynamics that are of interest). Because there are two measurement positions, there are two possible correlations using the ROTH processor, which are from sensor 1 to sensor 2 and vice versa. Note that for the ROTH processor between sensors 1 and 2 , $W_{x_{2} x_{1}}(\omega)=S_{x_{1} x_{2}}(\omega) / S_{x_{1} x_{1}}(\omega)$, and between sensors 2 and 1 , $W_{x_{2} x_{1}}(\omega)=S_{x_{2} x_{1}}(\omega) / S_{x_{2} x_{2}}(\omega)$. The modulus and phase of the two ROTH processors are calculated for the data from the test rig and are shown in Fig. 5(ai, aii) and (bi, bii) as solid blue lines respectively. In Fig. 5(ai) it can be seen that a resonance appears in modulus of the FRF between sensors 1 and 2, but in the FRF between sensors 2 and 1 an anti-resonance and a resonance peaks are evident in Fig. 5(bi). The deviation of the phase from straight-line behaviour associated with leak noise is also clear in both FRFs shown in Fig. 5(aii) and (bii). Note that the only difference between the phase plots is one of sign.

As mentioned previously, the parameters for the resonators need to be known if the dynamic effects due to the resonance behaviour are to be removed from the data. These parameters include the natural frequency and damping ratio. The natural fre-

(ai)

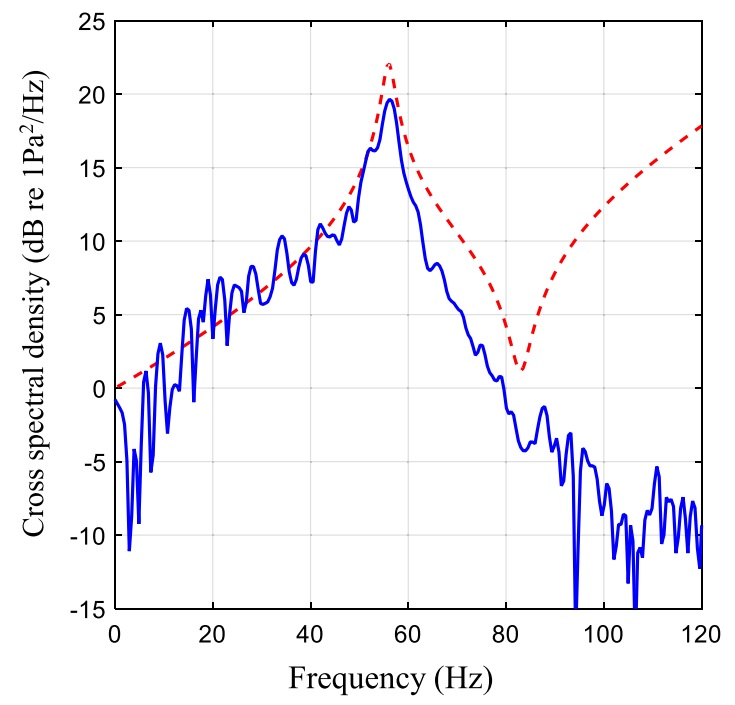

(bi)

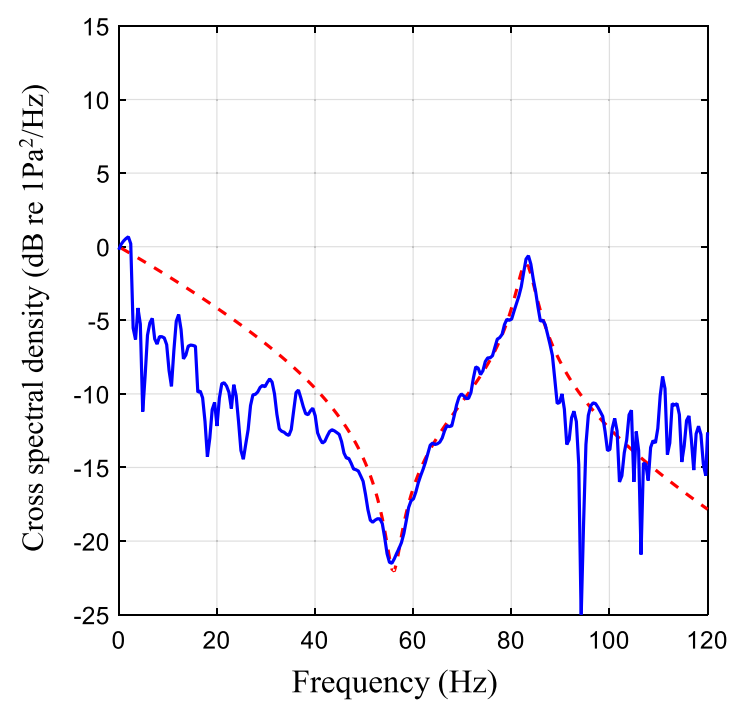

(aii)
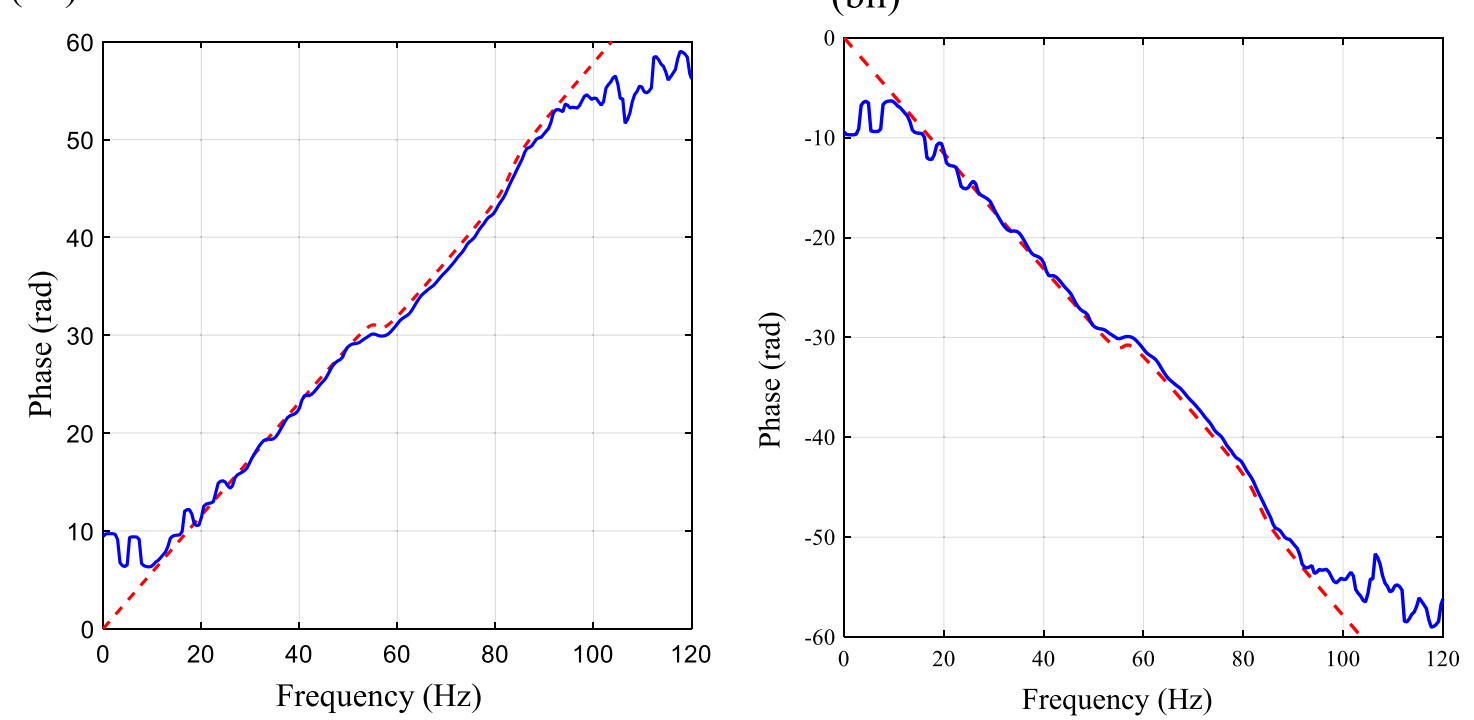

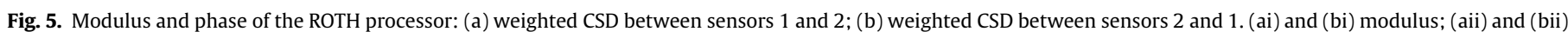

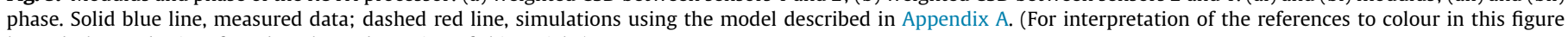
legend, the reader is referred to the web version of this article.) 
quencies can be determined directly by inspection of the PSDs, and the damping can be determined by curve fitting measured data to a model of the ROTH correlator at frequencies close to the resonance frequencies. Such a model, including the effects of the resonators, is given in Appendix A. Setting the natural frequencies of the resonators for sensor 1 and sensor 2 to be $56 \mathrm{~Hz}$ and $83 \mathrm{~Hz}$ respectively, the damping was found to be $\zeta=0.022$ for each resonator. The model results are overlaid with the measured data in Fig. 5. It can be seen that the model captures the modulus of the ROTH correlator between sensors 2 and 1, in Fig. 5(bi), but it does not fare as well for the measurement between sensors 1 and 2, in Fig. 5(ai). The reason for this is that the signals are dominated by high noise levels in the PSD at Position 1 above about $90 \mathrm{~Hz}$. The predicted modulus is much larger at higher frequencies in Fig. 5(ai) than in Fig. 5(bi), and is not captured in the measurements because of the high level of incoherent noise. Note that the model captures the behaviour of the phase well in both cases, as can be seen in Fig. 5(aii) and (bii).

(ai)

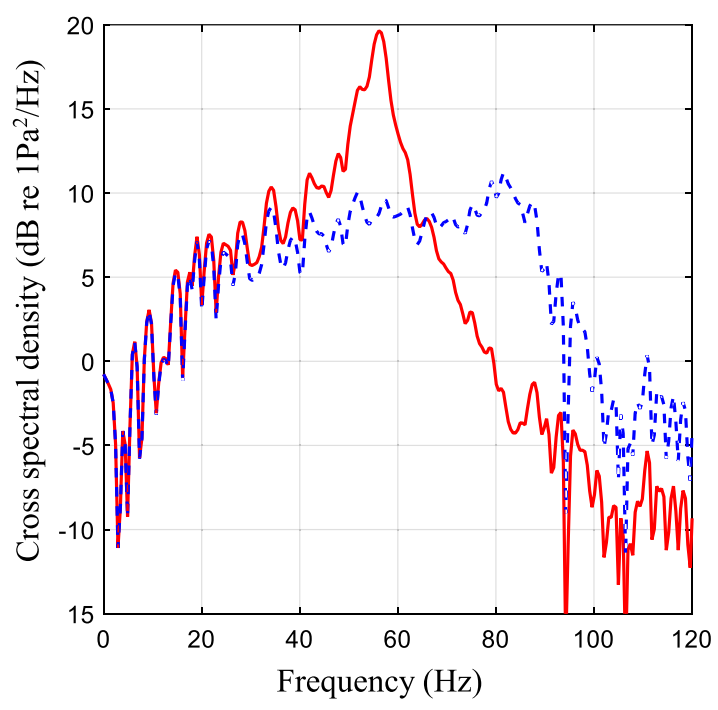

(aii)

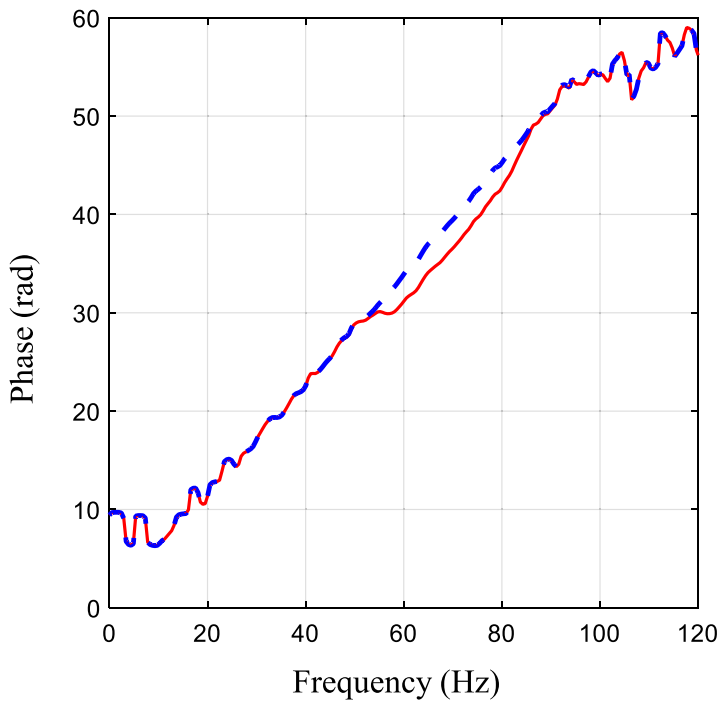

Once the parameters of the resonators have been determined, the effects of the resonances can be removed from the measured data by simply multiplying $W(\omega)$ in Eq. (2) by $D_{1} / D_{2}$ for $S_{x_{1} x_{2}}(\omega)$ and by $D_{2} / D_{1}$ for $S_{x_{2} x_{1}}(\omega)$ with respect to the two ROTH processors. The resulting CSDs for the ROTH correlators are shown in Fig. 6 as a dashed blue line. Also in this figure are the measured data shown in Fig. 5. It is clear from Fig. 6(ai) and (bi) that the peak and the trough are "flattened" in the moduli, giving smoother curves. Also, more importantly, the deviations from the phase due to the resonance effects are removed, as clearly evident in Fig. 6(aii) and (bii).

\section{Cross-correlation functions for TDE}

The correlation results for the BCC, PHAT and ROTH correlators are shown in Fig. 7(ai-aiv) for the raw data, and in Fig. 7(bi-biv) for the data processed so that resonance effects are removed. The dashed vertical line shows the true time delay of $92 \mathrm{~ms}$, assuming

(bi)

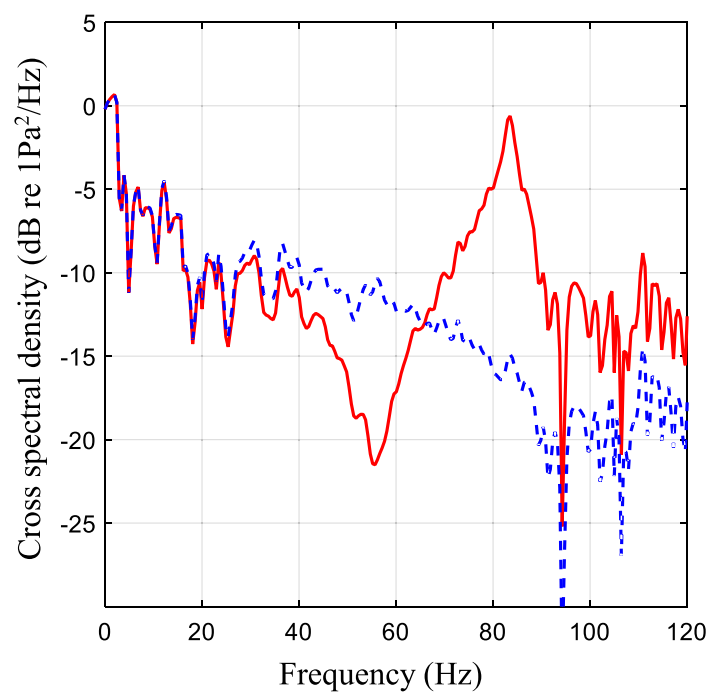

(bii)

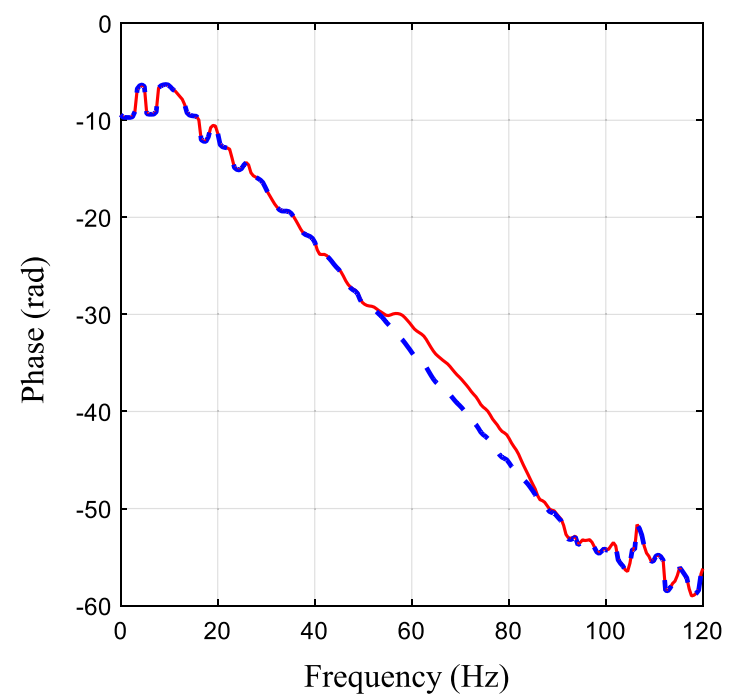

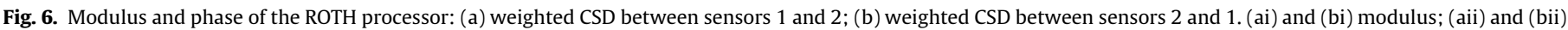

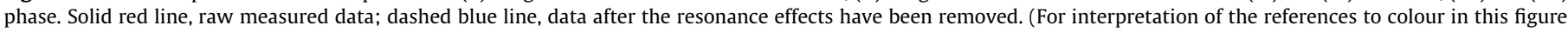
legend, the reader is referred to the web version of this article.) 
(ai)

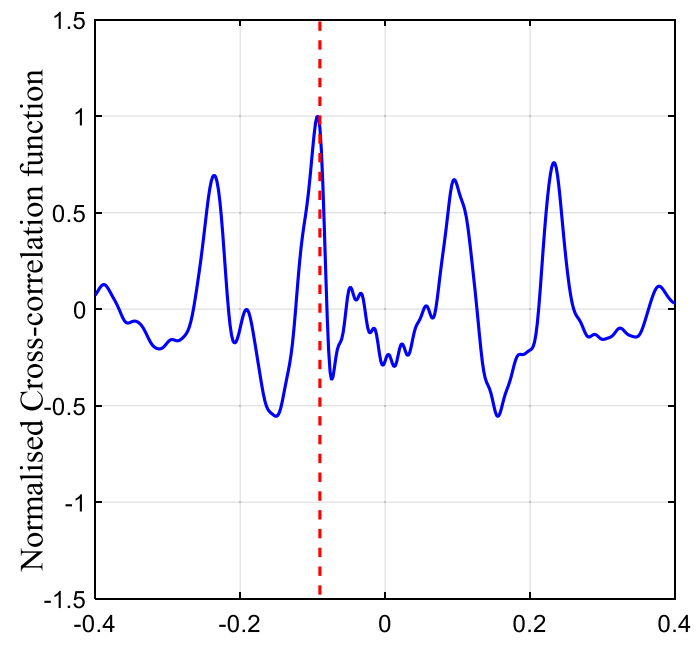

(aii)

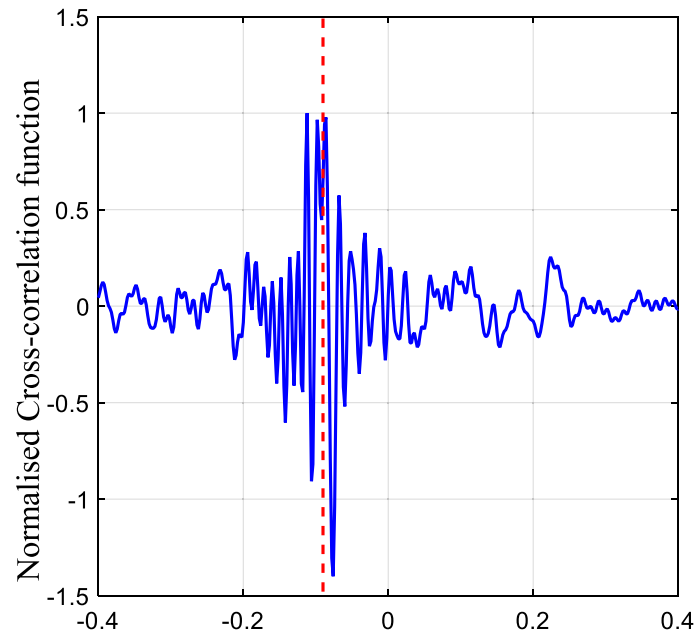

(aiii)

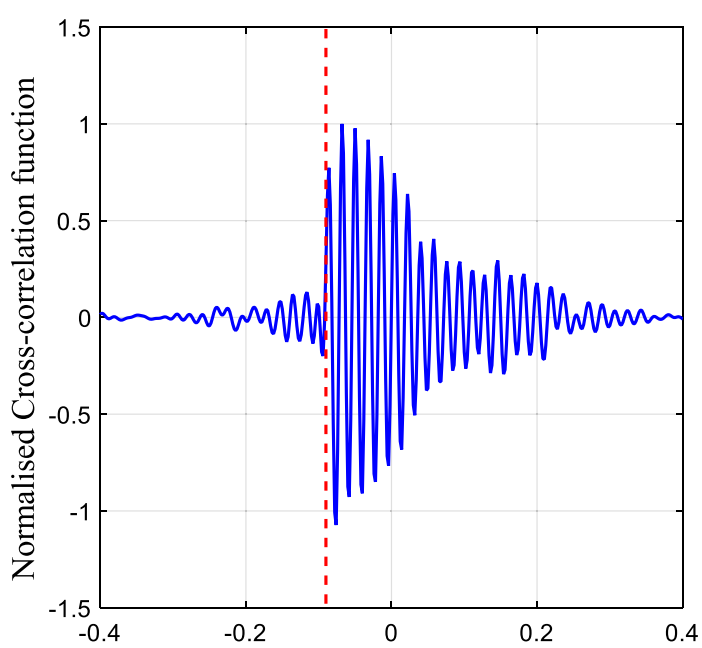

(bi)

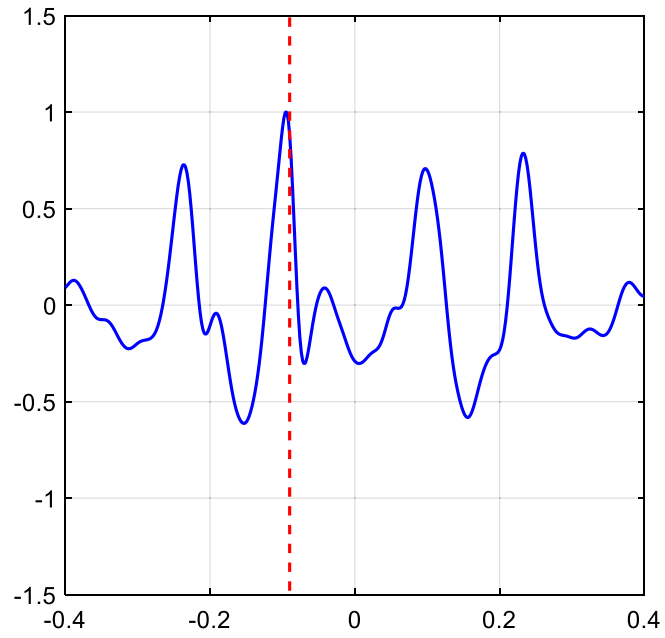

(bii)

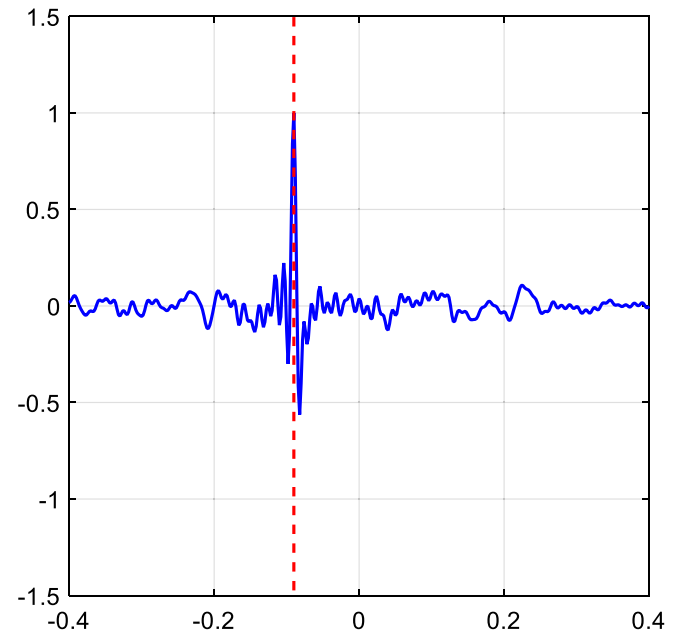

(biii)

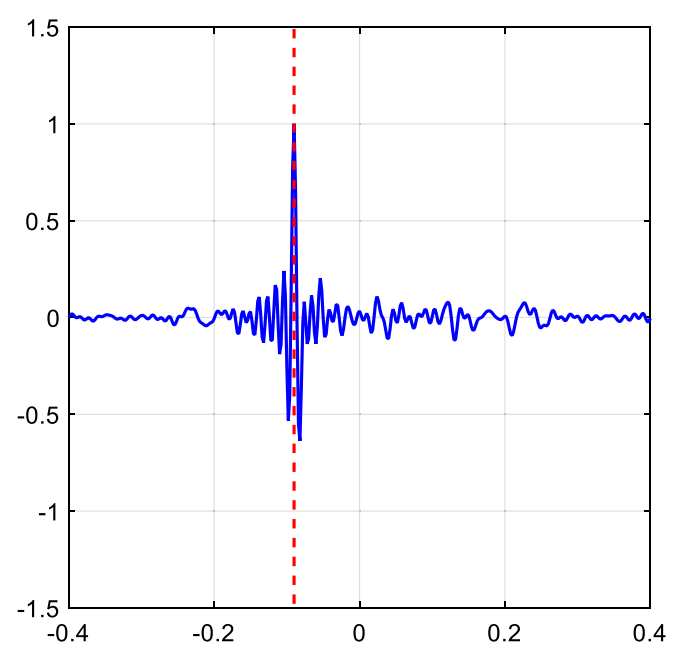

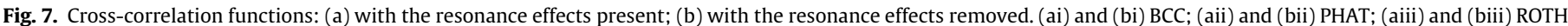
between sensors 1 and 2; (aiv) and (biv) ROTH between sensors 2 and 1 . The dashed vertical line corresponds to the true time delay of 92 ms. 
(aiv)

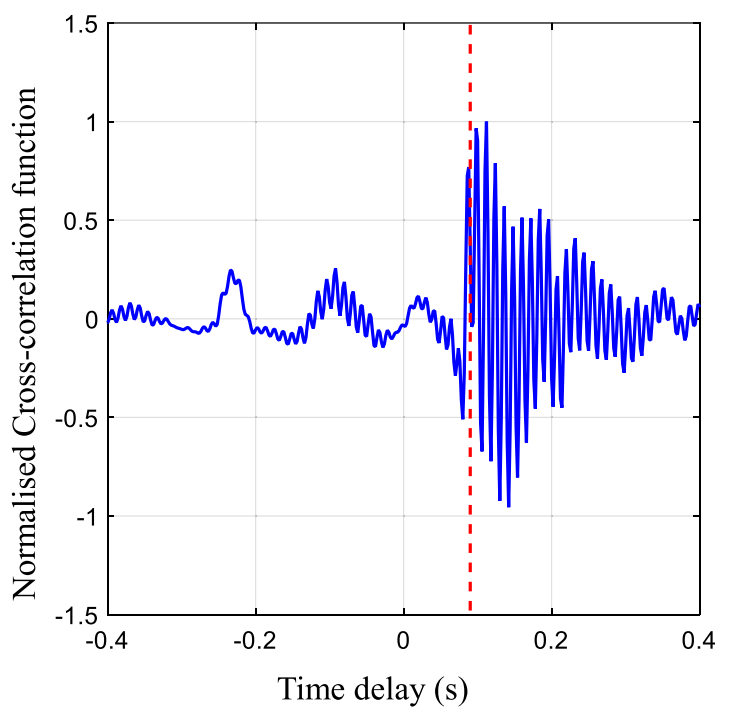

(biv)

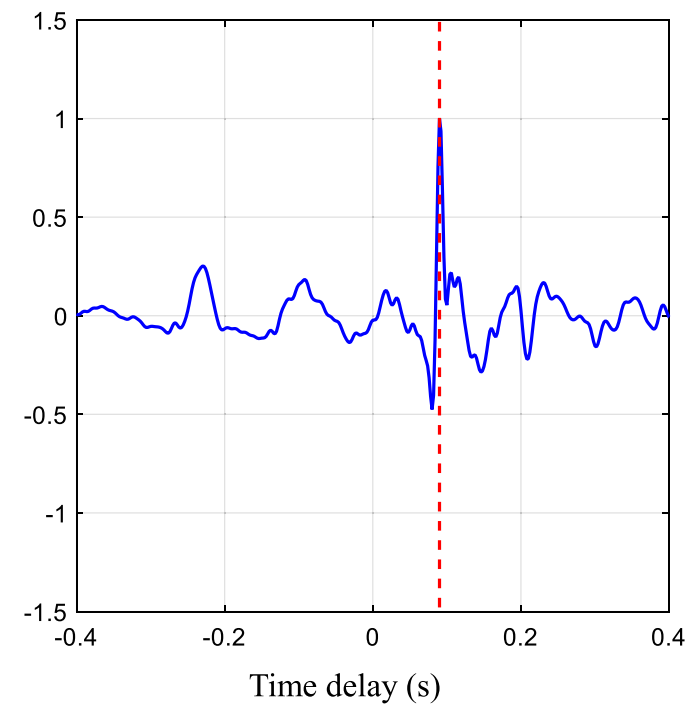

Fig. 7 (continued)

a wave speed of $482 \mathrm{~m} / \mathrm{s}$ calculated based on the measurements from an in-bracket leak source [2]. Consider first the BCC correlator without and with processing, which is respectively shown in Figs. 7 (ai) and (bi). The peak related to the time delay in both the figures is $94 \mathrm{~ms}$, a percentage difference of about $2 \%$. The additional peaks in both figures correspond to the reflections at the ends of the pipes at the measurement positions. These reflections are important at low frequencies because of the small attenuation of waves at these frequencies, and this phenomenon is discussed in detail in [12]. They manifest themselves strongly in the BCC function because of the spectral characteristics of the modulus of the CSD shown in Fig. 4(b), which weights low frequencies much more than high frequencies. The resonance effects do not have a large effect on the BCC function, but can be seen as additional ripples in Fig. 7(ai) compared with Fig. 7(bi).

Consider now the PHAT correlation results without and with processing, which is respectively shown in Fig. 7(aii) and (bii). It is clear that the PHAT correlator does not give the correct time delay if the bandwidth $5-90 \mathrm{~Hz}$ is chosen as shown in Fig. 7(aii), but it does give a good estimate of $90 \mathrm{~ms}$ if the resonance effects are removed as can be seen in Fig. 7(bii). Furthermore, as shown in Fig. 7(bii), the PHAT correlator also suppresses the additional peaks due to the reflections, removing some ambiguity in the interpretation of a cross-correlation function. The reason for this is that it gives equal weighting to low and high frequency data within the bandwidth chosen for analysis. Note that PHAT correlator can give a good estimate of the time delay without the removal of the resonance effects, but this can only be achieved when the bandwidth over which the analysis is conducted is restricted to low frequencies, below the first resonance [8].

For completeness, the ROTH correlation function between sensors 1 and 2 is shown in Fig. 7(aiii) and (biii). The correlation function without removing the resonance effects is shown in Fig. 7(aiii). It can be seen that this appears to be an impulse response with time advance. The peak in this does not correspond to the time delay estimate. Removing the resonance effects effectively reduces the slowly decaying oscillations from the correlation function, leaving only a sharp peak that occurs at $90 \mathrm{~ms}$. Examining the modulus of the CSD for the ROTH correlator in Fig. 6(ai), shows that this increases with frequency up to about $80 \mathrm{~Hz}$, and thus weights the higher frequencies more than lower frequencies, which results in the sharp peak. Broadly similar results are seen for the ROTH correlation function between sensors 2 and 1 as shown in Fig. 7 (aiv) and (biv). The differences between the ROTH correlation functions with the resonance effects removed, which can be seen in Fig. 7(biii) and (biv), can be attributed to the respective moduli of the CSDs shown in Fig. 6(ai) and (bi). It can be seen that the weighting functions are different, with the ROTH correlation function between sensors 1 and 2 weighting the higher frequencies more that the ROTH correlation function between sensors 2 and 1 .

To aid interpretation into why the correlators have different cross-correlation function shapes, the weighting functions $|W(\omega)|$ for the four correlators considered (the BCC, PHAT and ROTH $(\times 2)$ ) are shown for the raw data in Fig. 8(a) and for the data with the resonance effects removed in Fig. 8(b). Note that the weighting functions for the raw data are simply the moduli of the crossspectra. Moreover, the phases are the same for all three correlators, and are shown in Fig. 6(aii) or (bii). Examining Fig. 8(a) and (b), it is clear why the BCC is not greatly affected when the resonances are improved. It is also clear why the ROTH processers benefit by the removal of the resonances as the weighting functions become "flatter" with frequency and so give broadly similar results to the PHAT correlator. However, it should be noted that there is a choice of which ROTH correlator to choose from, and depending on particular situations, it is possible that they will give different time delay estimates.

\section{Discussion}

By examining the results shown in the previous section, it is clear that with some simple processing of the data, it is possible to significantly improve the shape of the cross-correlation function, and hence aid interpretation of this in the determination of time delay and location of a leak. This does, however, involve a certain amount of expertise, in which the analyst is able to observe resonance behaviour in the data. If this is possible then their effects can be removed using the procedure described in this paper (either one or more resonances), and the bandwidth over which the data can be used for TDE can be increased. Further, this allows the PHAT correlator to be used with confidence, over the bandwidth defined by good coherence, to remove the damping effects of the pipe (wave attenuation at high frequencies) and to suppress peaks 
(a)

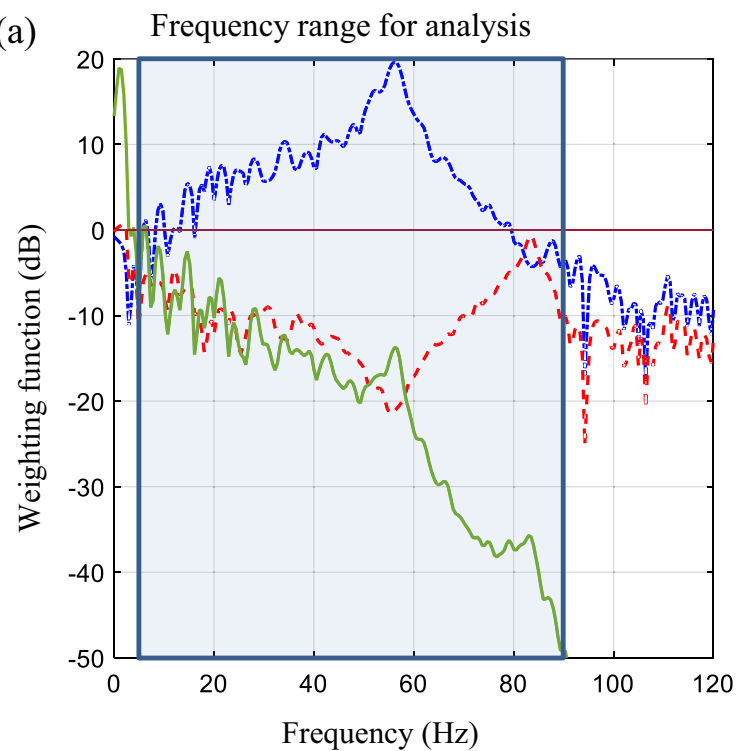

(b)

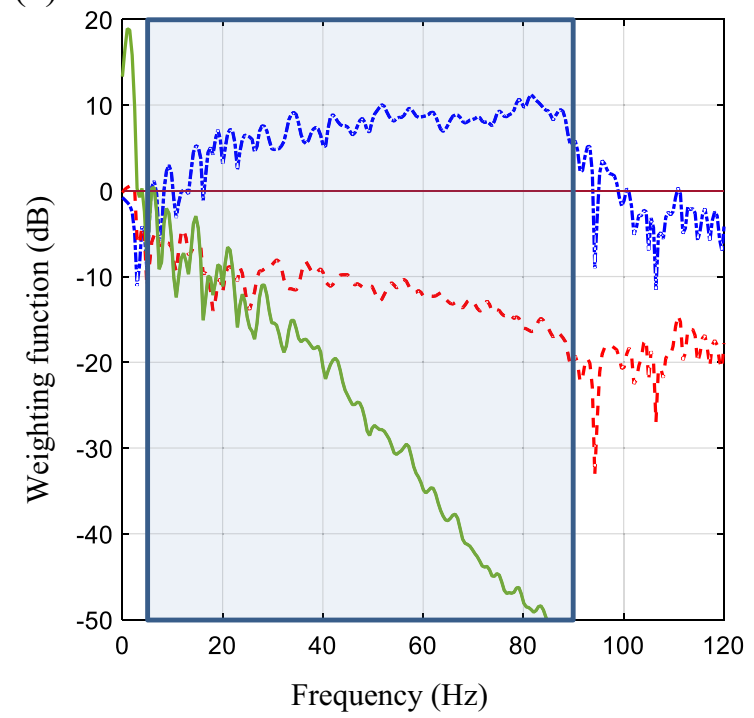

Fig. 8. Weighting functions $|W(\omega)|$ : (a) without the resonance effects removed; (b) with resonance effects removed. Solid green line, BCC; straight solid line at $0 \mathrm{~dB}$, PHAT; dashed-dotted blue line, ROTH between sensors 1 and 2; dashed blue line, ROTH between sensors 2 and 1 . (For interpretation of the references to colour in this figure legend, the reader is referred to the web version of this article.)

due to reflections, to give a clear and accurate estimate of the time delay as shown in Fig. 7(bii). It remains an open problem on how to determine the resonance effects in leak noise data automatically and remove them without human intervention.

\section{Conclusions}

In this paper, a way to improve the shape of the crosscorrelation function for leak detection in plastic water distribution pipes has been investigated. Using experimental data, it has been shown that significant improvement can be achieved by determining the FRFs for any resonators responsible for peaks in the CSD functions, and then processing the data using the FRFs of these resonators. The parameters of the resonators are best determined by examining the ROTH correlator as this is similar to the FRF of the system, and thus facilitates the identification of both resonances and anti-resonances if they exist. The net effect of processing the data is to remove any deviations of the phase from that expected for a pure time delay and to smooth the modulus of the CSD. Using the modified phase spectrum, the PHAT correlator can be used over the frequency bandwidth in which there is good coherence between the two measured signals, resulting in an unambiguous and clear estimate of the time delay. There are thus significant advantages in applying the process described in this paper. The current limitation, however, is that the procedure has to be applied manually, which will restrict its application in practical acoustic correlators. The challenge now is to develop an algorithm to carry out the procedure automatically.

\section{Acknowledgements}

The authors gratefully acknowledge the financial support of the CAS Hundred Talents Programme and FAPESP, project No. 2013/50412-3, and Osama Hunaidi from the National Research Council of Canada who provided the test data.

\section{Appendix A. Model for the ROTH correlator}

In this appendix, a model of the ROTH correlator is described. First, however, a model of leak noise propagation in an infinite pipe (with no resonators), is described. As the pipe is assumed to be of infinite length, there are no wave reflections from boundaries. The leak generates noise that propagates as a non-dispersive coupled plane wave involving the water and the pipe-wall away from the leak to the measurement positions [6,13]. The pressure of this wave at position $u$ is given by Ref. [6]

$p(\omega, u)=P(\omega) H(\omega, u)$

where $P(\omega)$ is the amplitude of the acoustic pressure at the leak location, and $H(\omega, u)=e^{-\omega \beta u} e^{-\mathrm{i} \omega u / c}$ is the FRF between the pressure at the leak position and the pressure at the measurement position, in which $u$ is the distance from the leak, $\beta$ is an attenuation factor and $c$ is the speed of wave propagation. For the pipe discussed in this paper $c$ is taken to be $482 \mathrm{~m} / \mathrm{s}$ and $\beta$ is taken to be $1.58 \times 10^{-4} \mathrm{~s} / \mathrm{m}$. Note that these are taken to be constants. Recent work by Gao et al. $[14,15]$ has shown that for plastic water pipes, the presence of the surrounding soil increases both the propagation wavespeed and attenuation with frequency compared to the invacuo case. However, they may be assumed to be constant for the purposes of this paper, because in the experimental work leak noise signals are confined to low frequencies.

The CSD between the two measured signals is given by Ref. [6]

$S_{x_{1} x_{2}}(\omega)=S_{l l} H^{*}\left(\omega, d_{1}\right) H\left(\omega, d_{2}\right)$

where $*$ denotes the complex conjugate; $S_{l l}$ is the PSD of the leak, which is assumed to be constant in the frequency range of interest; and the distances refer to those given in Fig. 1. The PSDs of the leak signals are given by

$S_{x_{1} x_{1}}(\omega)=S_{l l}\left|H\left(\omega, d_{1}\right)\right|^{2} ;$

$S_{x_{2} x_{2}}(\omega)=S_{l l}\left|H\left(\omega, d_{2}\right)\right|^{2}$

respectively. Noting that $W(\omega)=\Psi(\omega) S_{x_{1} x_{2}}(\omega)$ from Section 2, where $\Psi(\omega)$ is given in Table 1, the CSD for the ROTH correlator between sensors 1 and 2 and sensors 2 and 1 are respectively given by

$W_{x_{1} x_{2}}(\omega)=e^{\omega \beta\left(d_{1}-d_{2}\right)} e^{-j \omega T_{0}}$

$W_{x_{2} x_{1}}(\omega)=e^{-\omega \beta\left(d_{1}-d_{2}\right)} e^{-j \omega T_{0}}$

To include the effects of the resonances for the ROTH correlator between sensors 1 and $2, W_{x_{1} x_{2}}(\omega)$ is simply multiplied by $D_{2} / D_{1}$ where $D_{i}$ is the FRF of a resonator given by Eq. (4), and the sub- 
scripts denote sensors 1 and 2 respectively. To include the effects of the resonances for the ROTH correlator between sensors 2 and $1, W_{x_{2} x_{1}}(\omega)$ is multiplied by $D_{1} / D_{2}$.

\section{References}

[1] Fuchs HV, Riehle R. Ten years of experience with leak detection by acoustic signal analysis. Appl Acoust 1991:33:1-19.

[2] Hunaidi O, Chu WT. Acoustical characteristics of leak signals in plastic water distribution pipes. Appl Acoust 1999;58:235-54

[3] Bendat JS, Piersol AG. Engineering of applications of correlation and spectral analysis. 2nd ed. New York: Wiley; 1993.

[4] Gao Y, Brennan MJ, Joseph PF. A comparison of time delay estimators for the detection of leak noise signals in plastic water distribution pipes. J Sound Vib 2006:292:552-70.

[5] Brennan MJ, Gao Y, Joseph PF. On the relationship between time and frequency domain methods in time delay estimation for leak detection in water distribution pipes. J Sound Vib 2007;304:213-23.

[6] Gao Y, Brennan MJ, Joseph PF, Muggleton JM, Hunaidi O. A model of the correlation function of leak noise in buried plastic pipes. J Sound Vib 2004; $277: 133-48$.

[7] Almeida FCL, Brennan MJ, Joseph PF, Whitfield S, Dray S, Paschoalini A. On the acoustic filtering of the pipe and sensor in a buried plastic water pipe and its effect on leak detection: an experimental investigation. Sensors 2014;14:5595-610.

[8] Almeida FCL. Improved acoustic methods for leak detection in buried plastic water distribution pipes [PhD Thesis]. University of Southampton; 2013.

[9] Roth PR. Effective measurements using digital signal analysis. IEEE Spectr 1971;8:62-70

[10] Almeida FCL, Brennan MJ, Joseph PF, Dray S, Whitfield S, Paschoalini AT. Towards an in-situ measurement of wave velocity in buried plastic water distribution pipes for the purposes of leak location. J Sound Vib 2015;359:40-55

[11] Hunaidi O, Chu W, Wang A, Guan W. Detecting leaks in plastic pipes. J Am Water Works Ass 2000;92:82-94

[12] Gao Y, Brennan MJ, Joseph PF. On the effects of reflections on time delay estimation for leak detection in buried plastic water pipes. J Sound Vib 2009;325(3):649-63.

[13] Pinnington RJ, Briscoe AR. Externally applied sensor for axisymmetrical waves in a fluid-filled pipe. J Sound Vib 1994;173:503-16.

[14] Gao Y, Sui F, Muggleton JM, Yang J. Simplified dispersion relationships for fluid-dominated axisymmetric wave motion in buried fluid-filled pipes. J Sound Vib 2016;375:386-402.

[15] Gao Y, Liu Y, Muggleton JM. Axisymmetric fluid-dominated wave in fluid-filled plastic pipes: loading effects of surrounding elastic medium. Appl Acoust 2017;116:43-9. 